Human Ethology Bulletin 30 (2015)4: 23-31

Brief Report

\title{
ACTIVATION OF SELF-PROTECTION THREAT INCREASES WOMEN'S PREFERENCES FOR DOMINANCE IN MALE FACES
}

\author{
Donald F. Sacco ${ }^{1}$, Christopher Lustgraaf ${ }^{1}$, Mitch Brown ${ }^{1}$ \& Steven G. Young ${ }^{2}$ \\ ${ }^{1}$ Department of Psychology, Owings-McQuagge Hall, University of Southern Mississippi, \\ USA \\ ${ }^{2}$ Department of Psychology, Baruch College, City University of New York, USA \\ Donald.Sacco@usm.edu
}

\begin{abstract}
Women's self-reported vulnerability to aggression has been linked to a stronger preference for male body types communicating physical formidability and aggressive dominance. This preference shift is considered an adaptive trade-off whereby women in more dangerous environments increase their preference for men with a greater capacity to protect them and their offspring, even though such men are also more likely to be coercive toward them as a mate. The current study extends these findings in two critical ways. First, we primed women with a self-protection threat, versus a control experience, to determine if acute activation of vulnerability to aggression results in an increased preference for physically dominant men. Second, we utilized male faces that varied in the extent to which they communicate dominance and trust to determine if preferences for physical formidability and aggressive dominance extend to face preferences. Women exposed to a video which activated self-protection concerns displayed a stronger preference for male faces communicating high dominance (versus high trust) compared to women in a control video condition. These results suggest that women's preferences for dominance in men coincides with acutely activated self-protection threat, and also extends to dominance cues in faces.
\end{abstract}

Keywords: Face perception, evolution, self-protection, dominance 


\section{INTRODUCTION}

Contending with threats to physical safety, including those posed by other humans, was likely a recurring challenge in human evolutionary history. However, while guarding against aggressive others is a universal concern, women are relatively more vulnerable to violence from male conspecifics due to sexual dimorphism (i.e., larger size and greater muscle mass in men relative to women) and greater overall male aggressiveness (e.g., Daly \& Wilson, 1988; Frayer \& Wopoff, 1985). Given that aggression related to homicide, sexual assault, and resource competition were likely common in ancestral environments, it has been hypothesized that susceptibility to violence was an integral factor in shaping female mating preferences (Buss \& Schmitt, 1993).

In environments characterized by elevated levels of violence and group conflict, aggressive dominance may be an adaptive advantage for some men, facilitating access to resources. Consequently, women may have evolved a context-dependent mating strategy in which they demonstrate a preference for aggressive and formidable men in environments characterized by high levels of violence. However, this preference may represent an important trade-off for women. Specifically, aggressive and dominant men can inflict physical harm on female partners, and may be more likely to do so than less aggressive men (Sell, Cosmides, \& Tooby, 2009). Nonetheless, women in these dangerous environments likely faced an elevated risk of violence toward themselves and their offspring. The benefits of recognizing, and subsequently procuring, a more aggressive, dominant, and physically formidable male partner (i.e., a mate who could provide increased access to resources and protection from extra-pair violence) may have been greater than the costs of preferring such a male partner and facing increased exposure to potential physical danger.

To test the hypothesis that women's perceived vulnerability to aggression might result in elevated preferences for physically formidable and dominant men, Snyder and colleagues (2011; Study 3), primed women with either dangerous (e.g., a war-scarred urban street) or safe scenes (e.g., children playing in a public park) to differentially activate fear of violence. Participants then completed a fear of crime questionnaire and selected the body type of their ideal long-term mate from a matrix of body types that varied in fat and muscularity. Although the between-subjects manipulation did not result in differential body-type preferences across conditions, women's fear of crime was positively correlated with their preference for more physically formidable male body types (i.e., body types associated with aggressive dominance). These findings provide initial evidence that women possessing a greater dispositional perceived vulnerability to violence (e.g., crime) display an increased preference for aggressive-dominant males.

We sought to extend these findings in two critical ways. First, we utilized a well-validated method for activating self-protection motives by having female participants watch one of two videos-one which activated self-protection threat and a control video (Maner et al., 2005; Young, Slepian, \& Sacco, 2015). Although Snyder and colleagues (2011) found no impact of situationally activated self-protection concerns on women's dominance 
preferences, we suspected that a more validated manipulation of self-protection threat might provide evidence for this phenomenon. Specifically, we hypothesized that women primed with the self-protection threat video would demonstrate a stronger preference for dominant and physically formidable men compared to women primed with a control (nonthreatening) video.

Second, we asked women to view pairs of faces that varied in perceived dominance and trustworthiness based on facial morphological features. Importantly, these two traits are extracted rapidly from faces, and comprise fundamental components of perception and trait judgments (e.g., Oosterhof \& Todorov, 2008). Specifically, participants saw two versions of a male face: one manipulated to communicate high levels of dominance and the other manipulated to communicate high levels of trust. Because characteristics of trust and dominance may be valuable traits that women prefer in men, and because women were required to choose a face communicating only high levels of one of these traits, women's decisions would represent a trade-off between preferring dominance versus trust in male faces. That is, if women in the self-protection condition selected a dominant face instead of a trustworthy face, it suggests that under conditions of self-protection threat valuations of traits may shift in favor of dominance over trustworthiness, thus providing clear evidence that self-protection enhances the value of male dominance when women are considering a partner. Consistent with Snyder and colleagues' (2011) findings, we hypothesized that women in the self-protection condition would more frequently prefer a face communicating dominance over a face communicating trust, compared to control condition participants.

\section{METHOD}

\section{Participants}

Eighty-three women volunteered to participate in exchange for partial course credit. Due to computer error, data was not recorded for the dominance preference task (see description below) for 7 participants, resulting in a final sample of 76 participants (mean age $=21.51$ years, $S D=6.34$ years $)$.

\section{Materials}

Video Primes. The self-protection clip (duration of 1 minute and 34 seconds) included scenes from Silence of the Lambs in which a female FBI agent is stalked by a serial killer. The control clip (duration of 1 minute and 40 seconds) was from the film Koyaanisqatsi, which includes videography of urban living (e.g., people going up and down an escalator) and is accompanied by orchestral music. Importantly, both clips are well-validated, as the former has been demonstrated to elicit high levels of fear while the latter has been used as an effective control video in past research (e.g., Maner et al., 2005; Young et al., 2015). 
Manipulation Check. We utilized a 5-item scale to assess participants' emotional reactions to the video clips to ensure that the videos induced the anticipated emotional responses. Participants were instructed to "Please take a moment to reflect on the movie clip you watched earlier and answer the questions based on how you feel after watching the movie clip." Using a 7-point scale ( $1=$ not at all; $7=$ very $m u c h$ ), participants responded to the statements, "How fearful do you feel?," "How happy do you feel?," "How anxious do you feel?," and "How interested do you feel?" We chose these specific emotions because past research assessing acute activation of self-protection motives has demonstrated that they result in heightened self-reported feelings of fear, anxiety/tension, and interest, as well as reduced self-reported happiness (Maner et al., 2005). Additionally, participants responded to the statement, "Currently my mood is..." (1=very negative; $4=$ neutral; $7=$ very positive). We included this last item to demonstrate domain-specificity in emotional reactions to the self-protection prime; that is, we thought it prudent to demonstrate that the self-protection prime activates specific negative emotions, rather than results in greater general negative affect.

Dominance Preference Task. We utilized a computer-generated set of male stimuli which varied in the extent to which each face communicated levels of trust and dominance, respectively (see Oosterhof \& Todorov, 2008 for details regarding the creation and validation of this stimulus set). On each trial, participants were shown two male faces: one face was manipulated to exhibit high levels of trust, while the other face was manipulated to exhibit high levels of dominance; target identity was held constant on each trial, and face location was counterbalanced between-participants. Participants were tasked with selecting which face they preferred more ("which face do you prefer") using the left- and right-hand control keys on the keyboard, respectively. We utilized stimuli at two levels of extremity: 1 standard deviation away from the mean for both the trust and dominance prototypes, as well as 3 standard deviations away from the mean for both the trust and dominance prototypes (see fig. 1 for sample stimuli). Thus, decisions on trials using stimuli manipulated to be 3 standard deviations more extreme than the prototype for trust and dominance would represent a more extreme trade-off. We chose to use two levels of stimulus extremity to determine if women had a boundary, whereby preferring dominance over trust was potentially too costly; that is, women primed with self-protection may be willing to select a dominant face over a trustworthy face at low levels of stimulus extremity, but may be less willing to prefer a dominant face over a trustworthy face at higher stimulus intensity. Participants completed 16 trials of the task: 8 trials for 1 SD stimuli and 8 trials for 3 SD stimuli (pairs were always of matched stimulus intensity).

\section{Procedure}

After obtaining informed consent, participants were randomly assigned to one of two conditions on a between-participants basis. Specifically, participants either watched a video to activate self-protection threat concerns $(\mathrm{N}=39)$, or watched a control video $(\mathrm{N}=37)$. 
After watching the video, participants completed the manipulation check questionnaire followed by the trust-dominance preference task. Finally, participants completed a brief demographics form (including age and gender), were thanked for their participation and were debriefed.

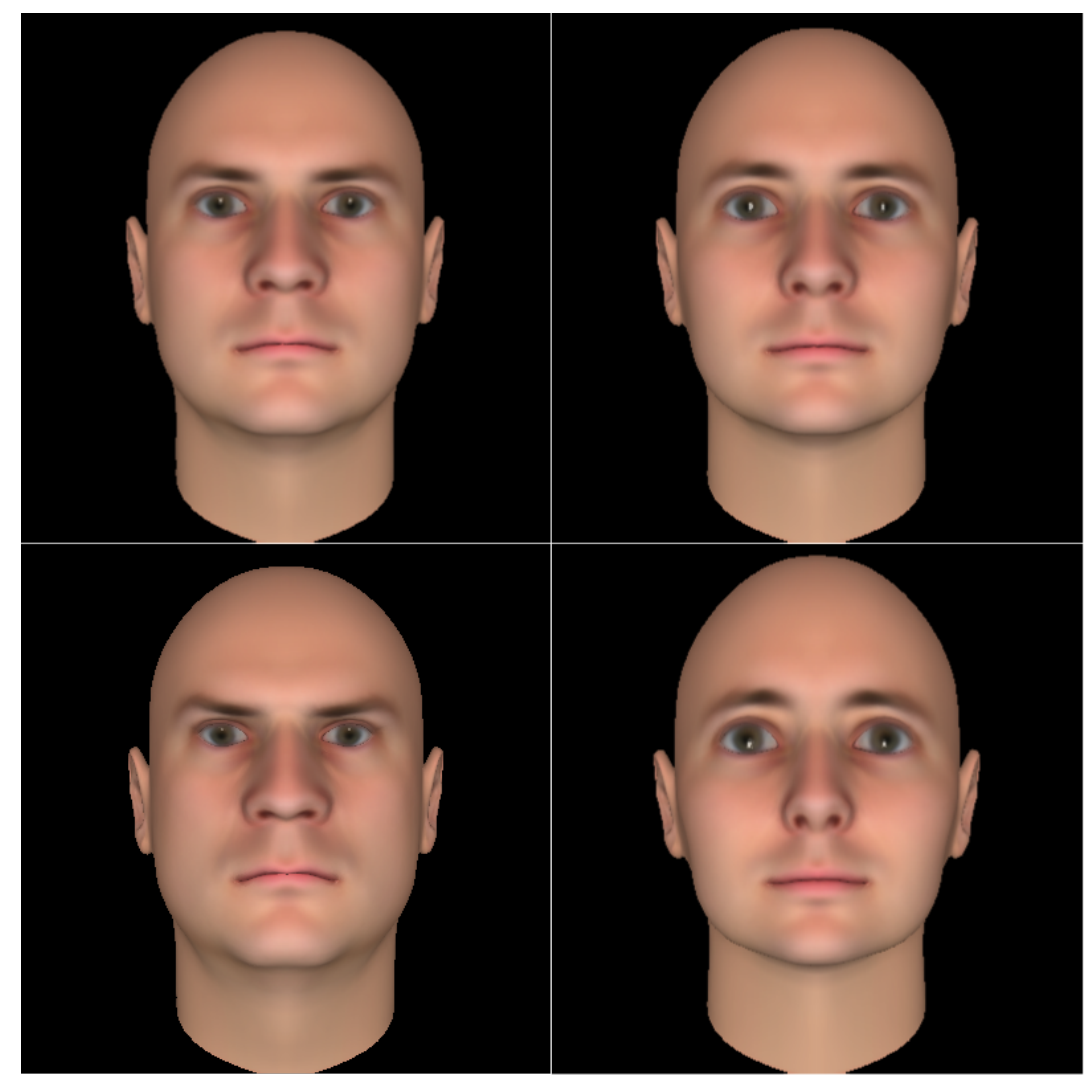

Figure 1: Sample dominance and trust stimuli. Row 1, Left is a sample low intensity dominant face; Row 1, Right is a sample low intensity trust face; Row 2, Left is a sample high intensity dominant face; Row 2, Right is a sample high intensity trust face.

\section{RESULTS}

\section{Manipulation Check}

Participants in the self-protection condition $(M=3.97, S E=.33)$ reported significantly more fear than participants in the control condition $(M=2.86 ; S E=.29), t(74)=2.53, p=.013$, $d=.58$. Participants in the self-protection condition $(M=1.95, S E=.20)$ reported significantly less happiness than control participants $(M=3.38 ; S E=.22), t(74)=-4.80, p<.001, d=1.10$. While participants in the self-protection condition did not report more anxiety $(M=4.67$, $S E=.31)$ than control participants $(M=4.46, S E=.28), t(74)=.50, p=.619, d=.35$, the means 
were in the predicted direction. Participants in the self-protection condition reported significantly greater interest $(M=5.15, S E=.28)$ than participants in the control condition $(M=3.65, S E=.29), t(74)=3.77, p<.001, d=.87$. Additionally, those in the self-protection condition reported similar overall $\operatorname{mood}(M=4.26, S E=.26)$ as control participants $(M=4.27$, $S E=.19), t(91)=-.043, p=.966, d=.01$. Consistent with past research (Maner et al., 2005), the video prime generally had the intended effect of inducing a self-protection mindset, as indexed by women in the self-protection condition reporting significantly greater fear and less happiness than women in the control condition. Additionally, participants across the self-protection and control conditions did not report different levels of general negativity, suggesting that the self-protection prime activated domain-specific emotions associated with self-protection threat.

\section{Dominance Preferences}

To determine whether the self-protection prime elicited different preferences for facial dominance compared to the control prime, we first computed participants' dominance preference at each of the two levels of stimulus intensity. Specifically, we divided the number of times participants selected the more dominant face by the total number of trials at each level of stimulus intensity ( 8 trials at each level of stimulus intensity). Thus, higher values are indicative of more frequent selection of a dominant face relative to a trustworthy face. We then conducted a 2 Condition (Self-protection, Control) x 2 Stimulus Intensity (1 SD, 3 SD) mixed model ANOVA with repeated measures over the second factor. ${ }^{1}$ This analysis yielded a main effect of stimulus intensity, $F(1,74)=37.58, p<.001, \eta_{p}{ }^{2}=.337$ (see fig. 2 ); not surprisingly, women displayed a stronger preference for dominance for low intensity stimuli $(M=.18, S E=.02)$ relative to high intensity stimuli $(M=.08, S E=.02)$. This is sensible, as there are both benefits (interpersonal protection) and drawbacks (interpersonal abuse) associated with dominance characteristics; thus, showing a stronger preference at lower intensities may be a way to balance the benefits and drawbacks of this trade-off.

This analysis also yielded a main effect of condition, $F(1,74)=8.14, p=.006, \eta_{p}{ }^{2}=.099$. Consistent with our predictions, women in the self-protection condition demonstrated a stronger preference for dominant male faces $(M=.18, S E=.03)$ compared to participants in the control condition $(M=.07, S E=.03)$. Although these values suggest that participants were unlikely to prefer dominant faces relative to trustworthy faces overall, this finding is consistent with previous research demonstrating that trustworthiness is of primary concern when individuals are judging others (Lewis \& Weigert, 1985). Importantly, preferences for

\footnotetext{
${ }^{1}$ When we controlled for changes in participant mood (fear, happiness, anxiety, interest, current mood) stemming from the viewing the videos, none of the mood items were significant covariates in the model (all ps >.21); additionally, although the main effect of stimulus intensity was no longer significant when controlling for changes in participant mood $(p=.815)$, the critical main effect of condition was still significant $(p=.018)$. Thus, although participants' self-reported mood was predictably influence by which video they viewed, these self-reported changes in mood do not seem to be responsible for the hypothesized differences in facial dominance preferences across conditions.
} 
dominance were significantly greater than zero across both the self-protection, $t(38)=5.29$, $p<.001, d=1.69$, and control conditions, $t(37)=4.51, p<.001, d=1.44$, indicating that the current results do not suffer from floor effects.

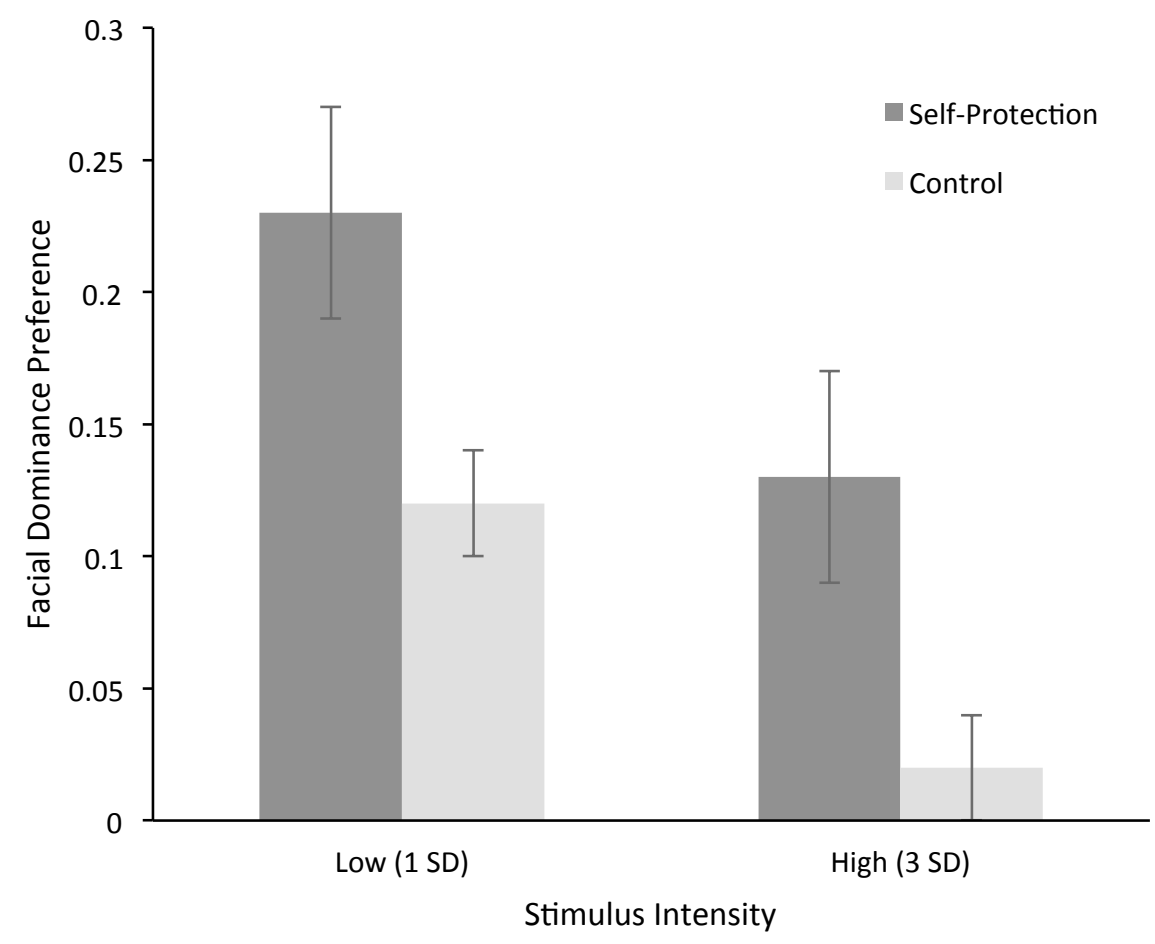

Figure 2: Dominance preferences across condition and stimulus intensity (Error bars represent the standard error of the mean).

Interestingly, the interaction between stimulus intensity and condition was not significant, $F(1,74)=.187, p=.666, \eta_{p}{ }^{2}=.003$; thus, for low intensity stimuli, participants in the selfprotection condition reported a larger dominance preference $(M=.23, S E=.04)$ than participants in the control condition $(M=.12, S E=.02), t(58.83)=2.80, p=.007, d=.64$, and for high intensity stimuli, participants in the self-protection condition also reported a larger dominance preference $(M=.13, S E=.04)$ than participants in the control prime condition $(M=.02, S E=.02), t(54.24)=2.51, p=.015, d=.58$ (because of unequal variance, we conducted corrected t-tests and report adjusted degrees of freedom, $t$-statistics and significance values). 


\section{DISCUSSION}

The results of the current study supported the primary hypotheses. Specifically, activating acute self-protection threat concerns translated into differences in facial dominance preferences across the two experimental conditions. Women primed with self-protection threat demonstrated a stronger preference for dominant male versus trustworthy male faces. In other words, when forced to choose between two potentially valuable traits (dominance and trust), activated self-protection threat led women to favor dominance over trust more often than women in the control prime condition. While recent research has linked situationally activated self-protection threat with enhanced discrimination of important social signals communicated in faces (e.g., Young et al., 2015), the current results demonstrate that self-protection threat concerns also influence preferences for facially communicated social traits, specifically trust versus dominance.

Interestingly, the extremity of this trade-off between trust and dominance did not seem to deter women in the self-protection condition from demonstrating a stronger preference for dominance in male faces compared to women in the control condition. That is, even for the more extreme versions of the stimuli used in this study (i.e., having to choose between an extremely dominant-looking male face versus an extremely trustworthy-looking male face), women primed with self-protection indicated an increased preference for dominant males across both stimulus extremities compared to women in the control condition. Although one might argue that there would be limits to such a trade-off, it may be the case that when threatened, a trade-off favoring dominance over trust might be adaptive for women, regardless of the extremity of such a trade-off.

The current findings add to a growing literature on context-dependent trade-offs in women's mating preferences. For example, in environments where health risks are high, women prefer more masculine men (a trade-off whereby women prioritize the production of healthy offspring over the cost of low male investment communicated by more masculine men; DeBruine, Jones, Crawford, Welling, \& Little, 2010). Additionally, women who are at peak fertility levels prefer men who display dominance and intrasexual competitiveness as a short-term mate more than women who are not at peak fertility (a trade-off favoring good genes over investment potential; Gangestad, Simpson, Cousins, Garver-Apgar, \& Christensen, 2004). Consistent with these findings, the current study found that acute activation of self-protection threat leads women to display a stronger preference for dominance rather than trust in male partners (compared to a control experience). In essence, women under self-protection threat prioritize a partner who can offer interpersonal protection, even though this partner may inflict violence on her (Snyder et al., 2011), and even at the expense of a more trustworthy partner. 


\section{REFERENCES}

Buss, D., \& Schmitt, D. (1993). Sexual strategies theory: An evolutionary perspective on human mating. Psychological Review, 100, 204-232. DOI

Daly, M., \& Wilson, M. (1988). Homicide. New York: Aldine de Gruyter.

DeBruine, L. M., Jones, B. C., Crawford, J. R., Welling, L. L., \& Little, A. C. (2010). The health of a nation predicts their mate preferences: Cross-cultural variation in women's preferences for masculinized male faces. Proceedings of the Royal Society of London B: Biological Sciences, 277, 2405-2410. DOI

Frayer, D. W., \& Wolpoff, M. H. (1985). Sexual dimorphism. Annual Review of Anthropology, 14, 429-473. DOI

Gangestad, S. W., Simpson, J. A., Cousins, A. J., Garver-Apgar, C. E., \& Christensen, P. N. (2004). Women's preferences for male behavioral displays change across the menstrual cycle. Psychological Science, 15, 203-207. DOI

Lewis, J.D., Weigert, A. (1985). Trust as a social reality. Social Forces, 63, 967-985. DOI

Maner, J. K., Kenrick, D. T., Becker, D. V., Robertson, T. E., Hofer, B., Neuberg, S. L., ... \& Schaller, M. (2005). Functional projection: How fundamental social motives can bias interpersonal perception. Journal of Personality and Social Psychology, 88, 63-78. DOI

Oosterhof, N. N., \& Todorov, A. (2008). The functional basis of face evaluation. Proceedings of the National Academy of Sciences, 105, 11087-11092. DOI

Sell, A., Tooby, J., \& Cosmides, L. (2009). Formidability and the logic of human anger. Proceedings of the National Academy of Sciences, 106, 15073-15078. DOI

Snyder, J. K., Fessler, D. M., Tiokhin, L., Frederick, D. A., Lee, S. W., \& Navarrete, C. D. (2011). Trade-offs in a dangerous world: Women's fear of crime predicts preferences for aggressive and formidable mates. Evolution and Human Behavior, 32, 127-137. DOI

Young, S. G., Slepian, M. L., \& Sacco, D. F. (2015). Sensitivity to perceived facial trustworthiness is increased by activating self-protection motives. Social Psychological and Personality Science, 6, 607-613. DOI 